\title{
Obtenção de corante natural de antocianinas extraídas de capim-gordura (Melinis minutiflora P. Beauv.) e estudo da aplicação em iogurtes
}

Obtaining natural dye from anthocyanins extracted from molasses grass (Melinis minutiflora P. Beauv.) and study of its application in yogurts

Obtención de colorantes naturales a partir de antocianinas extraídas de gordura (Melinis minutiflora P. Beauv.) y estudio de su aplicación en yogures

\section{Resumo}

O consumo de corantes artificiais vem preocupando os consumidores, o que impulsiona as pesquisas com corantes naturais e sua aplicação em alimentos. As antocianinas são uma classe de compostos com potencial para a utilização como corantes alimentícios, devido à sua abundância na natureza, extensa gama de cores e efeitos benéficos à saúde, com comprovada capacidade antioxidante. O capim-gordura (Melinis minutiflora), por sua vez, é uma fonte promissora de antocianinas, devido ao seu baixo custo e à presença de antocianinas metiladas em sua composição. $\mathrm{O}$ presente trabalho teve por objetivo a obtenção de um corante natural a partir de antocianinas de capim-gordura e sua aplicação em iogurtes. A extração das antocianinas de capim-gordura foi otimizada por meio de um Delineamento Composto Central Rotacional (DCCR) de duas variáveis, 8 ensaios e três pontos centrais, onde foram definidas as condições ótimas de $15 \mathrm{~g}$ de capim para $50 \mathrm{~mL}$ de solução extratora, com 38,5\% de etanol. Os extratos obtidos a partir das condições ótimas foram rotaevaporados para a retirada do etanol e liofilizados, obtendo-se no final um corante concentrado. Foram obtidos dois tipos de iogurtes, com e sem a aplicação de corante e avaliados quanto ao pH, acidez, capacidade de retenção de água e coloração durante 27 dias de armazenamento. Os iogurtes demostraram boa estabilidade ao armazenamento, com redução na intensidade de cor a níveis não perceptíveis a olho nu. Os resultados demostraram o potencial da utilização de corante natural de capim-gordura para esse tipo de matriz alimentícia.

Palavras-chave: Antocianinas metiladas; Corantes alimentícios; Delineamento composto central rotacional; Estabilidade.

\begin{abstract}
The consumption of artificial dyes has been worrying consumers, driving research on natural dyes and their application in food. Anthocyanins are a class of compounds with potential for use as food coloring, due to their abundance in nature, wide range of colors and beneficial health effects, with proven antioxidant capacity. Molasses grass (Melinis minutiflora) is a promising source of anthocyanins due to its low cost and the presence of methylated anthocyanins in its composition. The objective of the present work was to obtain a natural dye from molasses grass anthocyanins and its application in yogurts. The extraction of anthocyanins from molasses grass was optimized by a Central Composite Rotational Design (DCCR) with two variables, eight trials and three central points, where the optimal conditions of $15 \mathrm{~g}$ of grass to $50 \mathrm{~mL}$ of extracting solution, with $38.5 \%$ ethanol. The extracts obtained from the optimal conditions were rotaevaporated to remove the ethanol and lyophilized, getting a concentrated dye. Two types of yogurts were produced, with and without applying the dye and evaluated for $\mathrm{pH}$, acidity, water holding capacity and color during 27 days of storage. The yogurts showed good storage stability, reducing the color intensity
\end{abstract}


to levels not perceptible to the unaided human eye. The results showed the potential of using natural dye from molasses grass for this type of food matrix.

Keywords: Food coloring; Methylated anthocyanins; Rotational central composite design; Stability.

\section{Resumen}

El consumo de colorantes artificiales viene preocupando a los consumidores, lo que impulsa la investigación sobre colorantes naturales y su aplicación en alimentos. Las antocianinas son una clase de compuestos con potencial para su uso como colorante alimentario, debido a su abundancia en la naturaleza, amplia gama de colores y efectos beneficiosos para la salud, con capacidad antioxidante comprobada. El pasto gordura (Melinis minutiflora), a su vez, es una fuente prometedora de antocianinas, debido a su bajo costo y la presencia de antocianinas metiladas en su composición. El objetivo del presente trabajo fue obtener un colorante natural a partir de antocianinas de pastos gordura y su aplicación en yogures. Se optimizó la extracción de antocianinas de gordura utilizando un Diseño Rotacional Compuesto Central (DCCR) con dos variables, 8 pruebas y tres puntos centrales, donde se determinaron las condiciones óptimas de $15 \mathrm{~g}$ de pasto para $50 \mathrm{~mL}$ de solución extractora, con 38,5\% de etanol. Los extractos obtenidos de las condiciones óptimas fueron rotaevaporados para eliminar el etanol y liofilizados, obteniendo al final un colorante concentrado. Se obtuvieron dos tipos de yogures, con y sin aplicación de colorante y se evaluó su pH, acidez, capacidad de retención de agua y color durante 27 días de almacenamiento. Los yogures mostraron una buena estabilidad al almacenamiento, con una reducción en la intensidad del color a niveles no perceptibles a ojo humano. Los resultados mostraron el potencial del uso de colorante natural de pasto gordura para este tipo de matriz alimentaria.

Palabras clave: Antocianinas metiladas; Colorante alimenticio; Diseño compuesto central rotacional; Estabilidad.

\section{Introdução}

A cor é um importante atributo sensorial nos alimentos, sendo associada pelos consumidores ao sabor, segurança e qualidade do produto (Pinela et al., 2019). A perda da cor natural dos alimentos durante o processamento e armazenamento ou a necessidade de incorporar características mais atrativas a um produto, são os grandes responsáveis pela adição de corantes pela indústria alimentícia (Albuquerque et al., 2020). Assim, os corantes alimentícios são substâncias naturais ou sintéticas utilizadas na conversão, restauração ou padronização da cor em alimentos (Backes et al., 2020).

Inicialmente, todos os corantes alimentícios eram de origem natural, retirado de plantas ou microrganismos. No entanto, com o advento da síntese de compostos a partir do século XIX, numerosos corantes sintéticos foram desenvolvidos e utilizados como aditivos alimentares (Amchova et al., 2015). Não obstante, alguns desses corantes sintéticos passaram a ser banidos devido a evidentes sinais de toxicidade causados por eles a curto e longo prazo.

Atualmente é crescente a preocupação acerca dos riscos de ingestão de corantes artificiais. É amplamente aceito que o consumo de corantes alimentares por crianças pode induzir a intolerância e reações alérgicas. Estudos recentes sugerem também o impacto deles na saúde dos adultos, sobretudo à característica carcinogênica de alguns deles, embora ainda com resultados pouco conclusivos (Feketea \& Tsabouri, 2017). Por conseguinte, é notória a preferência dos consumidores por corantes naturais em detrimento aos sintéticos, sob alegação de serem mais saudáveis e seguros para o consumo (Mojica et al., 2017).

A utilização de corantes naturais surge então como necessidade na indústria de alimentos, embora apresente certas dificuldades que necessitam ser contornadas. A estabilidade dos pigmentos naturais é de grande interesse dos pesquisadores, uma vez que a maioria deles é bastante reativa, tornando-se pouco estáveis às condições de processamento e composição química dos alimentos (Martins et al., 2016). Dentre os diversos pigmentos com potencial para a utilização na indústria de alimentos, é possível citar as antocianinas.

As antocianinas são uma classe de pigmentos com coloração que variam entre vermelho, azul e roxo. Representam o maior grupo de pigmentos fenólicos e o mais importante grupo de pigmentos hidrossolúveis de origem vegetal. Além de conferir cor, as antocianinas possuem efeitos benéficos à saúde, graças à sua ação antioxidante, com alegações de redução tanto do risco de doenças coronárias quanto de citotoxicidade, assim como efeitos anti-inflamatórios (Backes et al., 2020). 
A obtenção de antocianinas a partir de fontes naturais com menor custo, como resíduos de frutos ou outros biorresiduos, tem sido de grande interesse para a produção de corantes com valor agregado, sendo uma abordagem sustentável que permite atender às necessidades atuais de indústria e às demandas da sociedade, sem causar efeito na demanda por alimentos (Albuquerque et al., 2020). Assim, a extração de antocianinas a partir de matérias-primas não alimentícias apresenta vantagens em relação às fontes convencionais. Nesse contexto, o capim-gordura aparece como uma potencial fonte industrial de antocianinas.

O capim gordura (Melinis minutiflora P. Beauv.) é uma espécie perene nativa da África e introduzida no Brasil para fins de pastejo, sendo atualmente considerada uma planta invasora do Cerrado brasileiro. Bastante rústica e com alta produtividade, esse capim é utilizado na alimentação animal e na recuperação de áreas degradadas. Possui inflorescência na forma de panícula com coloração roxa, para a qual foram identificadas 21 estruturas de antocianinas, derivadas de cianidina com diferentes graus de metilação (Neves et al., 2021).

O iogurte, leite fermentado por cultivos proto-simbióticos de Streptococcus salivarius subsp. thermophilus e Lactobacillus delbrueckii subsp. Bulgaricus (Rodrigues et al., 2021), é um produto lácteo muito consumido e apreciado, sobretudo por crianças. Estima-se que no Brasil, 74\% da população consome iogurte, englobando um total de $76 \%$ da produção láctea do país (Ogliari \& Novello, 2021). Este produto é elaborado com a incorporação de diversos ingredientes adicionais, os quais conferem sabor ao produto, sendo também permitida a utilização de aditivos, incluindo corantes. A legislação brasileira permite a utilização de 20 tipos de corantes, dentre os quais estão alguns naturais e outros sintéticos (Brasil, 2007).

Neste contexto, o presente trabalho teve por objetivo a extração de antocianinas a partir do capim-gordura para elaboração de corante natural e aplicação em iogurtes, com avaliação da persistência da coloração ao longo do armazenamento.

\section{Metodologia}

\subsection{Matérias-primas e ingredientes}

As inflorescências de capim-gordura Melinis minutiflora P. Beauv., Poaceae foram coletadas em Diamantina-MG (18¹2’21" S; 4334'54” O) em junho de 2021, quando foi observada a maturação das suas inflorescências.

Para o extrato de antocianinas de capim gordura foi utilizado álcool de cereais Quimidrol (lote 42857). Todos os demais reagentes utilizados foram em grau analítico.

Para a elaboração dos iogurtes foi utilizado leite integral UHT, leite em pó desnatado, sacarose e cultura láctica tradicional YoFlex Mild 1.0 (Lactobacillus delbrueckii subsp. bulgaricus e Streptococcus thermophilus) (Chr. Hansen ${ }^{\circledR}$ ).

\subsection{Planejamento experimental}

Para a avaliação das condições ótimas de extração das antocianinas do capim-gordura foi realizado um Delineamento Composto Central Rotacional (DCCR) com 2 variáveis independentes, composto por 11 ensaios, onde foram testadas a influências da proporção de etanol e da proporção sólido/solvente sob a extração das antocianinas (Tabela 1). 
Tabela 1. Níveis codificados e reais das variáveis independentes estudadas no DCCR para extração de antocianinas em capimgordura.

\begin{tabular}{ccccc}
\hline \multirow{2}{*}{ Ensaio } & \multicolumn{2}{c}{ Níveis codificados } & \multicolumn{2}{c}{ Níveis reais } \\
\cline { 2 - 5 } & $\begin{array}{c}\text { Proporção de etanol } \\
(\% \text { v/v })\end{array}$ & Proporção sólido/solvente & $\begin{array}{c}\text { Proporção de etanol } \\
(\% \text { v/v })\end{array}$ & $\begin{array}{c}\text { Proporção } \\
\text { sólido/solvente }\end{array}$ \\
\hline 1 & -1 & -1 & 11,63 & 4,75 \\
2 & 1 & -1 & 68,36 & 4,75 \\
3 & -1 & 1 & 11,63 & 13,29 \\
4 & 1 & 1 & 68,36 & 13,29 \\
5 & 0 & 0 & 40 & 9 \\
6 & 0 & 0 & 40 & 9 \\
7 & 0 & 0 & 40 & 9 \\
8 & $-1,41$ & 0 & 0 & 9 \\
9 & 1,41 & 0 & 80 & 3 \\
10 & 0 & $-1,41$ & 40 & 15 \\
11 & 0 & 1,41 & 40 & \\
\hline
\end{tabular}

Fonte: Autores (2022).

Os 11 ensaios, sendo 4 fatoriais (combinações entre os níveis -1 e +1 ), 4 axiais (uma variável no nível $\pm \alpha$ e a outra 0) e 3 centrais (as duas variáveis no nível 0), foram realizados conforme descrito por Jaekel, Schmiele, \& Chang (2020). A Eq. 1 representa o modelo genérico da função de regressão.

$$
Y=\beta_{0}+\beta_{1} x_{1}+\beta_{2} x_{2}+\beta_{11} x_{1}^{2}+\beta_{22} x_{2}^{2}+\beta_{12} x_{1} x_{2}+\varepsilon
$$

Onde:

$\mathrm{Y}=$ variável dependente em estudo;

$\beta_{0}=$ constante de regressão;

$\beta_{1}, \beta_{2}$ e $\beta_{12}=$ coeficiente de regressão linear, quadrático e de interação, respectivamente;

$\mathrm{x}_{1}$ e $\mathrm{x}_{2}=$ valores codificados das variáveis independentes;

$\varepsilon=$ erro experimental

As demais condições de extração foram sonicação por 30 minutos em cuba de ultrassom Cristófoli (Campo Mourão, PR) com frequência de $42 \mathrm{KHz}$ e 30 minutos de agitação em mesa agitadora Solab SL180/DT (Piracicaba- SP) a 150 rpm. Essas condições foram estabelecidas com base em testes preliminares (dados não publicados). Além disso, o pH da solução extratora foi ajustado para 2,0 com adição de ácido láctico. Foi avaliada a concentração de antocianinas como variável resposta, sendo que a otimização objetivou obter a maior concentração de antocianinas no extrato com menor utilização de etanol.

\subsection{Análise de antocianinas totais}

$\mathrm{O}$ teor de antocianinas totais foi determinado pelo método do $\mathrm{pH}$ diferencial, conforme metodologia descrita por AOAC (2005). Alíquotas de $1 \mathrm{~mL}$ de cada extrato foram transferidas para dois balões volumétricos de $10 \mathrm{~mL}$. O primeiro balão foi completado com cloreto de potássio $(0,025 \mathrm{M}, \mathrm{pH} 1,0)$ e o segundo com acetato de sódio $(0,4 \mathrm{M}, \mathrm{pH} 4,5)$. Em seguida, foram realizadas leituras em espectrofotômetro (Bel Photonics UV-M51) para as duas soluções diluídas nos comprimentos de onda de 520 e 700nm. As concentrações de antocianinas dos extratos foram obtidas por meio da Eq. 2. 
Onde:

$\mathrm{A}=\left(\mathrm{Absorbância}_{520 \mathrm{~nm}}-\mathrm{AbS}_{700 \mathrm{~nm}}\right)_{\text {cloreto de potassio }}-\left(\mathrm{Abs}_{520 \mathrm{~nm}}-\mathrm{Abs}_{700 \mathrm{~nm}}\right)_{\text {Acetato de sódio }}$

\subsection{Preparo do corante}

Após definidas as condições ótimas de extração, foi elaborado um extrato que posteriormente foi rotaevaporado à vácuo $\left(55^{\circ} \mathrm{C}\right.$ e pressão de $\left.-600 \mathrm{mb}\right)$ para retirada o etanol e liofilizado $\left(-40^{\circ} \mathrm{C}\right.$, vácuo de $0,12 \mathrm{mb}$ por 48 horas) (Liofilizador de bancada Christ Alpha 2-4 LD Plus), obtendo-se ao final um corante concentrado. O corante foi armazenado em refrigerador $\left(4^{\circ} \mathrm{C} \pm 1\right)$ até o momento da utilização.

\subsection{Preparo de iogurte}

O iogurte do tipo batido foi elaborado a partir da dissolução a $40^{\circ} \mathrm{C}$ do leite em pó ao leite UHT integral. A adição de leite em pó foi calculada visando obter um leite com teor final de $10 \%$ de sólidos não gordurosos. Foi adicionada a sacarose e, em seguida, a mistura foi submetida a um tratamento térmico em banho-maria a $85^{\circ} \mathrm{C}$ por 30 minutos. A mistura foi resfriada até atingir $45^{\circ} \mathrm{C}$ para a inoculação da cultura láctica e posteriormente incubada a $45^{\circ} \mathrm{C}$ até que atingisse pH 4,6-4,5 (Rodrigues et al., 2021). Terminado o processo, o iogurte foi resfriado e dividido em duas amostras, para a qual, uma delas foi acrescida do corante obtido anteriormente $(4 \mathrm{~mL} / \mathrm{L})$. As duas amostras foram batidas e divididas em porções individuais, que ficaram armazenadas sob refrigeração durante 36 dias, durante os quais foram realizadas as análises de vida de prateleira. Os iogurtes foram preparados em triplicata, para cada tratamento.

\subsection{Caracterização dos iogurtes}

Após a elaboração, os iogurtes foram analisados quanto a cor, $\mathrm{pH}$, acidez total titulável, capacidade de retenção de água e atividade antioxidante. A cada três dias foram analisados a cor (somente para o iogurte acrescido de corante), pH e acidez e a cada seis dias foi analisada a capacidade de retenção de água. A seguir estão descritas as metodologias utilizadas nas análises.

\subsection{Análise de cor instrumental}

A coloração dos extratos foi determinada em espectrofotômetro Mini Scan XE 45/0-L (HunterLab, Reston, USA), nas seguintes condições de teste: iluminante D65 e ângulo de visão de $10^{\circ}$, com leitura direta dos valores das coordenadas $\mathrm{L}^{*}$ (luminosidade), $a^{*}\left(\right.$ intensidade de vermelho vs verde) e $b^{*}$ (intensidade de amarelo vs azul). Para a verificação da diferença entre a cor dos iogurtes ao longo do armazenamento, foi calculado $\Delta \mathrm{E}^{*}$ conforme a Eq. 3.

$$
\Delta E^{*}=\sqrt{\left(\Delta L^{*}\right)^{2}+\left(\Delta a^{*}\right)^{2}+\left(\Delta b^{*}\right)^{2}} \quad \text { Eq. } 3
$$

Onde:

$\mathrm{E}^{*}=$ variação total de cor;

$\mathrm{L}^{*}$ = variação do parâmetro $L^{*}$ (luminosidade);

$\mathrm{a}^{*}=$ variação do parâmetro $a^{*}$;

$\mathrm{b}=$ variação do parâmetro $b^{*}$. 


\subsection{Análise de pH e acidez titulável total}

$\mathrm{O} \mathrm{pH}$ foi determinado por leitura direta em potenciômentro digital, previamente calibrado com padrões de $\mathrm{pH}$ 4,0 e 7,0 conforme as especificações do Instituto Adolfo Lutz (IAL, 2008).

O teor de acidez titulável total foi quantificado segundo método titulométrico descrito pelo IAL (2008). Para tanto, foram utilizadas $5 \mathrm{~g}$ de amostra diluídas em $10 \mathrm{~mL}$ de água destilada e fenolftaleína como indicador.

\subsection{Capacidade de retenção de água (CRA\%)}

A capacidade de retenção de água dos iogurtes foi determinada submetendo-se uma amostra de 10 gramas em teste à centrifugação a 15000 x g por 15 minutos a $20^{\circ} \mathrm{C}$ (temperatura ambiente) (Harte, Luedecke, Swanson, \& Barbosa-Cánovas, 2003), sendo a capacidade calculada conforme a Eq. 4.

$$
\text { CRA }\left(C_{6}\right)=\left[1-\frac{\text { peso do soro apósa centrifugaghio }}{\text { peso da amostra }}\right] \times 100 \quad \text { Eq.4. }
$$

\subsection{Atividade antioxidante}

A atividade antioxidante foi medida por meio do teste de porcentagem de inibição da solução de DPPH, conforme metodologia descrita por Carvalho et al. (2020) adaptado.

Uma alíquota de $3 \mathrm{~g}$ de iogurte foi adicionada de $5 \mathrm{~mL}$ de solução de metanol (85\%), centrifugada a 2500rpm por 5 minutos e o sobrenadante, constituído pela solução de metanol contendo os compostos de ação antioxidante, foi recolhido para análise. De cada amostra, foram recolhidos $400 \mu \mathrm{L}$ e adicionados de $1600 \mu \mathrm{L}$ da solução de DPPH. Após 30 minutos de reação, foi feita a leitura a 517nm. Os resultados foram expressos em porcentagem de inibição (\%).

\subsection{Análises estatísticas}

Os dados obtidos no delineamento experimental foram avaliados por meio da Metodologia de Superfície de Resposta para cálculo dos coeficientes de regressão e análise de variância (ANOVA) com nível de significância de 10\%. O ponto otimizado foi determinado com base na metodologia proposta por Derringer e Suich (1980). Os dados da caracterização dos iogurtes foram comparados pelo teste $t$-Student $(\mathrm{p}<0,05)$ e as características avaliadas por tempo de armazenamento foram submetidas ao teste de regressão linear simples.

\section{Resultados e Discussão}

\subsection{Obtenção das condições ótimas de extração das antocianinas de capim-gordura}

Os resultados obtidos para a concentração das antocianinas totais nos ensaios estabelecidos pelo DCCR estão dispostos na Tabela 2. 
Tabela 2. Variável resposta (concentração de antocianinas) para os ensaios elaborados a partir do DCCR para a extração de antocianinas do capim-gordura.

\begin{tabular}{cc}
\hline Ensaio & $\begin{array}{c}\text { Concentração de antocianinas } \\
(\mathrm{mg} / \mathrm{L})\end{array}$ \\
\hline 1 & 40,74 \\
2 & 33,73 \\
3 & 80,32 \\
4 & 80,48 \\
5 & 55,61 \\
6 & 55,94 \\
7 & 71,47 \\
8 & 45,25 \\
9 & 28,22 \\
10 & 28,55 \\
11 & 90,17 \\
\hline
\end{tabular}

Fonte: Autores (2022).

A avaliação dos dados por meio da Metodologia de Superfície de Resposta $\left(R^{2}>0,85\right.$ e p-valor $\left.<0,10\right)$ permitiu observar que o termo linear da proporção de capim-gordura por solução extratora $\left(\beta_{2}=21,72 ; p<0,001\right)$ apresentou o melhor resultado para a extração dos compostos bioativos. Por outro lado, a porcentagem de etanol na solução extratora no termo quadrático desfavoreceu a extração do pigmento $\left(\beta_{1}=-10,02 ; \mathrm{p}=0,020\right)$, sendo que $88,14 \%$ dos dados experimentais foram explicados pelo modelo matemático. Tais resultados podem ser visualizados na Figura 1, onde a extração das antocianinas foi maximizada na maior proporção sólido/solvente e com a porcentagem de etanol no ponto central.

Figura 1. Gráfico de superfície de resposta para os parâmetros porcentagem de etanol da solução extratora e proporção sólido/solvente para ensaios envolvendo a extração de antocianinas de capim-gordura.

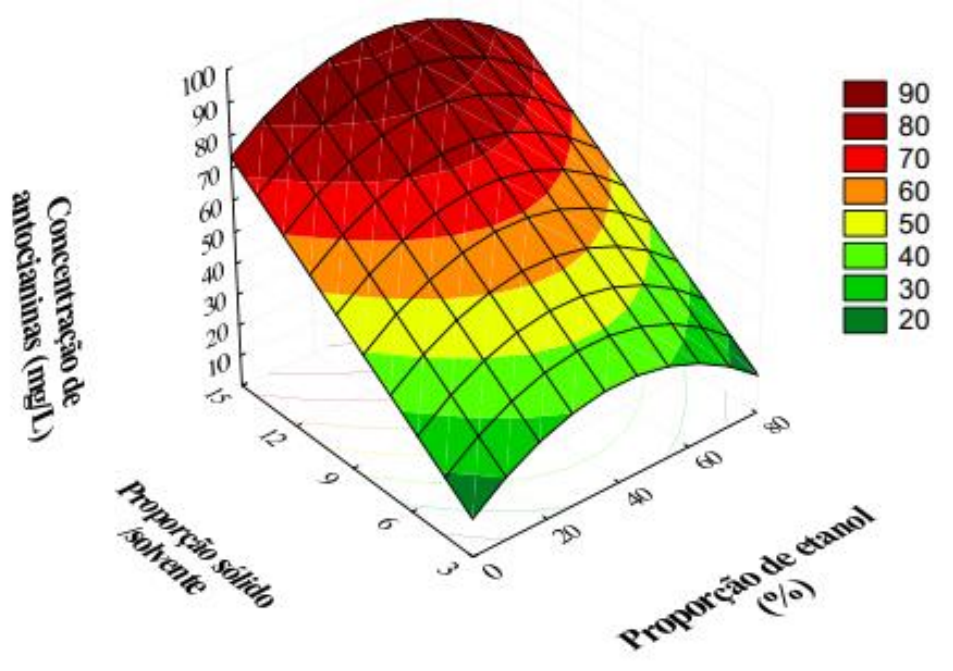

Fonte: Autores (2022).

A condição ótima de extração foi determinada através da otimização numérica, de forma a se obter os maiores valores preditos para o teor de antocianinas totais. Os resultados demostraram que a maior extração ocorreu com concentração de $38,58 \%$ de etanol/água $(\mathrm{v} / \mathrm{v})\left(\mathrm{x}_{1}=\mathrm{o}, 68\right)$ e proporção de $15 \mathrm{~g}$ de capim-gordura $\left(\mathrm{x}_{2}=+1,41\right)$ para $50 \mathrm{~mL}$ de solução extratora, sendo o valor predito de $93,31 \mathrm{mg}$ de antocianinas totais/L de extrato. A melhor condição de extração das antocianinas totais 
apresentou um grau de desejabilidade de 0,9044. O processo de validação do modelo matemático, realizado em triplicata verdadeira, foi obtido um valor experimental de 97,90 mg/g de antocianinas totais/L de extrato, resultando em um desvio relativo de 4,94\%. Diante destes resultados, o modelo matemático preditivo foi considerado válido, pois apresentou desvio relativo inferior a $10 \%$.

Essas condições de extração foram utilizadas para a obtenção do corante de capim-gordura empregado na elaboração dos iogurtes. Após a extração, a solução obtida foi rotaevaporada e submetida à liofilização, conforme descrito no item 2.4 da metodologia.

\subsection{Caracterização dos iogurtes}

Os iogurtes elaborados, com e sem corante, foram submetidos às análises de caracterização. Os resultados obtidos estão representados na Tabela 3 .

Tabela 3. Caracterização dos iogurtes padrão e colorido com corante obtido a partir de capim-gordura.

\begin{tabular}{cccc}
\hline Parâmetro & Iogurte Padrão & Iogurte com corante \\
\hline & $\mathrm{L}^{*}$ & $93,4 \pm 1,72$ & $96,2 \pm 0,08$ \\
Cor instrumental & $\mathrm{a}^{*}$ & $-8,12 \pm 0,55$ & $8,43 \pm 0,10$ \\
$\mathrm{nH}$ & $\mathrm{b}^{*}$ & $18,25 \pm 2,74$ & $2,42 \pm 0,01$ \\
Acidez titulável total (g de ácido lático/100g) & & $4,60 \pm 0,03$ & $4,22 \pm 0,02$ \\
Capacidade de retenção de água (\%) & $0,78 \pm 0,02$ & $1,02 \pm 0,01$ \\
Capacidade antioxidante (\% de inibição do radical DPPH) & $61,38 \pm 1,48$ & $57,86 \pm 0,99$ \\
\hline
\end{tabular}

Fonte: Autores (2022).

As duas formulações de iogurte, com e sem adição de corante, apresentaram valores estatisticamente diferentes para todos os parâmetros avaliados, de acordo com o teste t-Student com 5\% de intervalo de confiança.

As diferenças apresentadas na coloração dos iogurtes estão associadas à incorporação do corante obtido a partir do capim-gordura, rico em antocianinas, especialmente derivados metilados de cianidina-3-glicosídeo (Neves et al., 2021). Em pH ácido, o corante apresou coloração vermelha intensa, que, ao ser incorporado ao iogurte, resultou na coloração rosa, conforme demostrado na Figura 2. Por apresentar coloração rosa, o iogurte adicionado de corante apresentou maior valor para a coordenada $\mathrm{a}^{*}$, que sendo um valor positivo representa tons de vermelho.

Figura 2. a) Corante obtido a partir do capim-gordura, b) iogurte elaborado sem adição do corante, c) iogurte elaborado com a adição do corante.

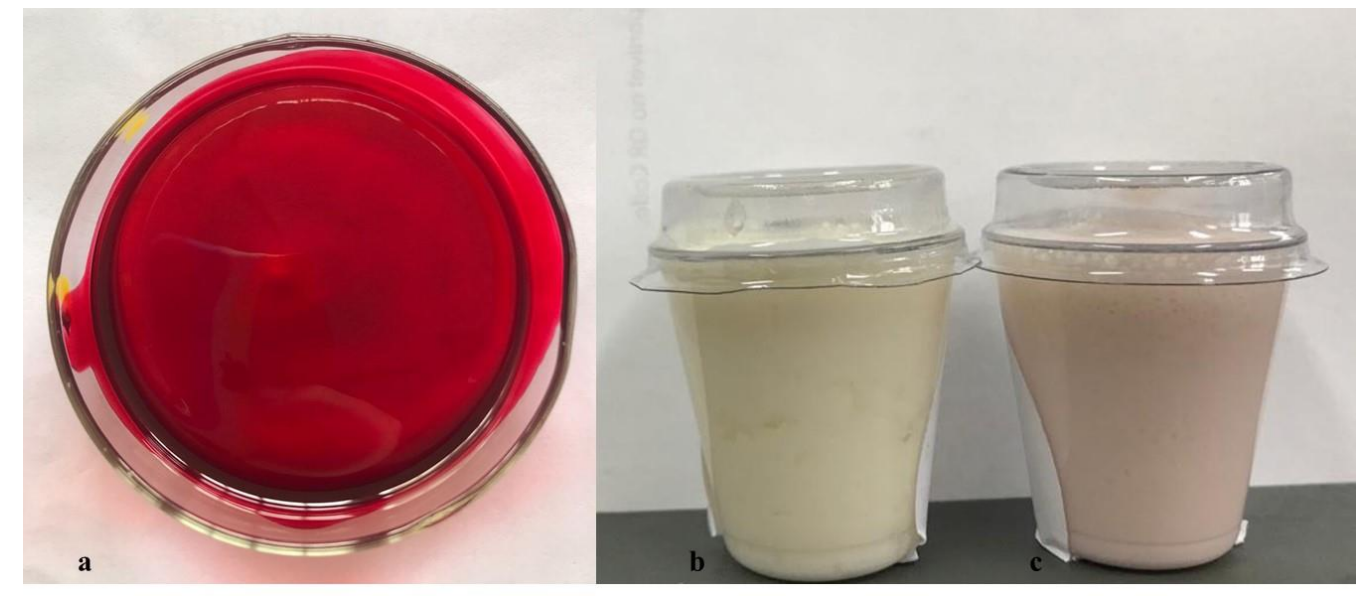

Fonte: Autores (2022). 
No processo de obtenção de iogurtes, as bactérias lácticas são responsáveis pela produção de ácido láctico a partir da fermentação da lactose, resultando em redução do pH do meio e, consequentemente, desnaturação das proteínas do leite, que causa a redução na repulsão eletrostática entre as micelas de caseína e a formação de géis, que confere a textura característica do produto (Batista et al., 2020). Assim, o valor de pH ideal para a formação de iogurtes é próximo a 4,5 (abaixo do ponto isoelétrico da caseína, 4,6) (Meng et al., 2018), no entanto, a legislação brasileira estabelece valores entre 3,6 e 4,5 (Brasil, 2007). Os valores de pH encontrados nos iogurtes elaborados estão em concordância com o preconizado na legislação. O iogurte adicionado de corante obteve menor valor de $\mathrm{pH}$, tal fato é justificado pela adição do corante, que, por ter sido obtido por extração com ácido láctico $(\mathrm{pH}=1,76)$, resultou na adição deste ao iogurte, fato confirmado pelo maior teor de acidez apresentado nesse iogurte. Valores semelhantes aos encontrados nesse trabalho foram obtidos por Gregório et al. (2020) para iogurtes do tipo grego adicionados de compota de carambola (4,25 e 4,60) e por Piovesan et al. (2021) para iogurtes do tipo frozen $(4,40$ a 4,49$)$.

A capacidade de retenção de água (CRA\%) em iogurtes pode ser entendida como a medida inversamente proporcional à sinérese. Por sua vez, a sinérese é o fenômeno de liberação espontânea de água do gel, sendo considerada um defeito, afetando negativamente a percepção da aparência do produto pelos consumidores (Rodrigues et al., 2021). Diversos fatores podem favorecer a sinérese em iogurtes, tais como alta temperatura de incubação na fermentação, alta concentração proteica, baixo teor de sólidos totais e alterações físicas do produto durante o armazenamento (Silva et al., 2012). A capacidade de retenção de água do iogurte batido tende a ser menor que os demais iogurtes, uma vez que o processo mecânico de bater o iogurte após a fermentação para incorporar novos ingredientes, tende a desestabilizar a rede tridimensional de micelas. Ainda assim, a capacidade de retenção de água encontrada para os iogurtes nesse trabalho foi maior que os valores encontrados em iogurtes adicionados de sucralose e colágeno (entre 29,67 e 35,50\%) (Rodrigues et al., 2021) e dentro da faixa dos valores encontrados por Vianna et al. (2019) em iogurtes elaborados a partir de leite de ovelha, cabra e vaca, em diferentes temperaturas de armazenamento (53,45 a 94,56\%). O iogurte sem corante apresentou maior capacidade de retenção de água do que o com o corante, fato que pode ser atribuído ao menor pH apresentado pela adição do corante.

Em relação à atividade antioxidante, notou-se um pequeno aumento da capacidade de inibição do radical DPPH no iogurte adicionado de corante. Embora adicionada em pequenas quantidades, as antocianinas possuem uma alta atividade antioxidante, podendo contribuir para o incremento dessa característica em produtos alimentícios. No entanto, a maior parte da capacidade antioxidante observada em ambos os iogurtes está relacionada à presença de peptídeos bioativos liberados durante a fermentação do leite por bactérias lácticas (Cho et al., 2020).

\subsection{Avaliação das características dos iogurtes durante o armazenamento}

Os iogurtes foram avaliados em intervalos de três dias até o $27^{\circ}$ dia, quando foi observado o surgimento de mofo em uma das embalagens, momento a partir do qual os produtos foram considerados impróprios para o consumo.

Na Figura 3 estão representados os valores de pH e acidez total titulável para os iogurtes com e sem corante, ao longo dos 27 dias de acompanhamento. Por meio dos dados apresentados, observou-se que a acidez de ambos os iogurtes tendeu a aumentar ao longo do tempo de armazenamento, com regressão significativa $(\operatorname{pr}(\mathrm{t})<0,05)$ para o iogurte com corante $\left(\mathrm{R}^{2}=\right.$ 0,4184) e para o sem corante $\left(\mathrm{R}^{2}=0,5314\right)$. Tal fato é esperado para iogurtes, fenômeno conhecido como pós-acidificação, e ocorre devido à manutenção da atividade metabólica das bactérias lácticas mesmo à temperatura de armazenamento, podendo ocorrer até pelo menos quatro semanas após a fabricação (Coelho et al., 2009; Silva et al., 2016). O fato descrito também explica a variação do $\mathrm{pH}$ ocorrida, no entanto com correlação significativa somente para o iogurte sem corante $\left(\mathrm{R}^{2}=0,6916\right)$. $\mathrm{A}$ menor variação da acidez e pH do iogurte com corante pode estar relacionada à maior acidez inicial dos iogurtes, devido ao acréscimo do corante. A presença de ácido láctico em maior concentração pode inibir o crescimento das bactérias lácticas, 
levando à manutenção do teor de acidez do iogurte (Coelho et al., 2009), ou, como no caso apresentado, somente à uma menor taxa metabólica. O aumento da acidez também é um indicativo indireto da ausência de contaminação do iogurte, uma vez que algumas espécies de bolores e leveduras utilizam o ácido láctico como substrato para o seu metabolismo, ocasionando na diminuição do pH dos iogurtes ao longo do armazenamento (Coelho et al., 2009).

Figura 3. Valores de pH e acidez total titulável para os iogurtes sem corante e com corante natural obtido a partir de capimgordura, ao longo do armazenamento.

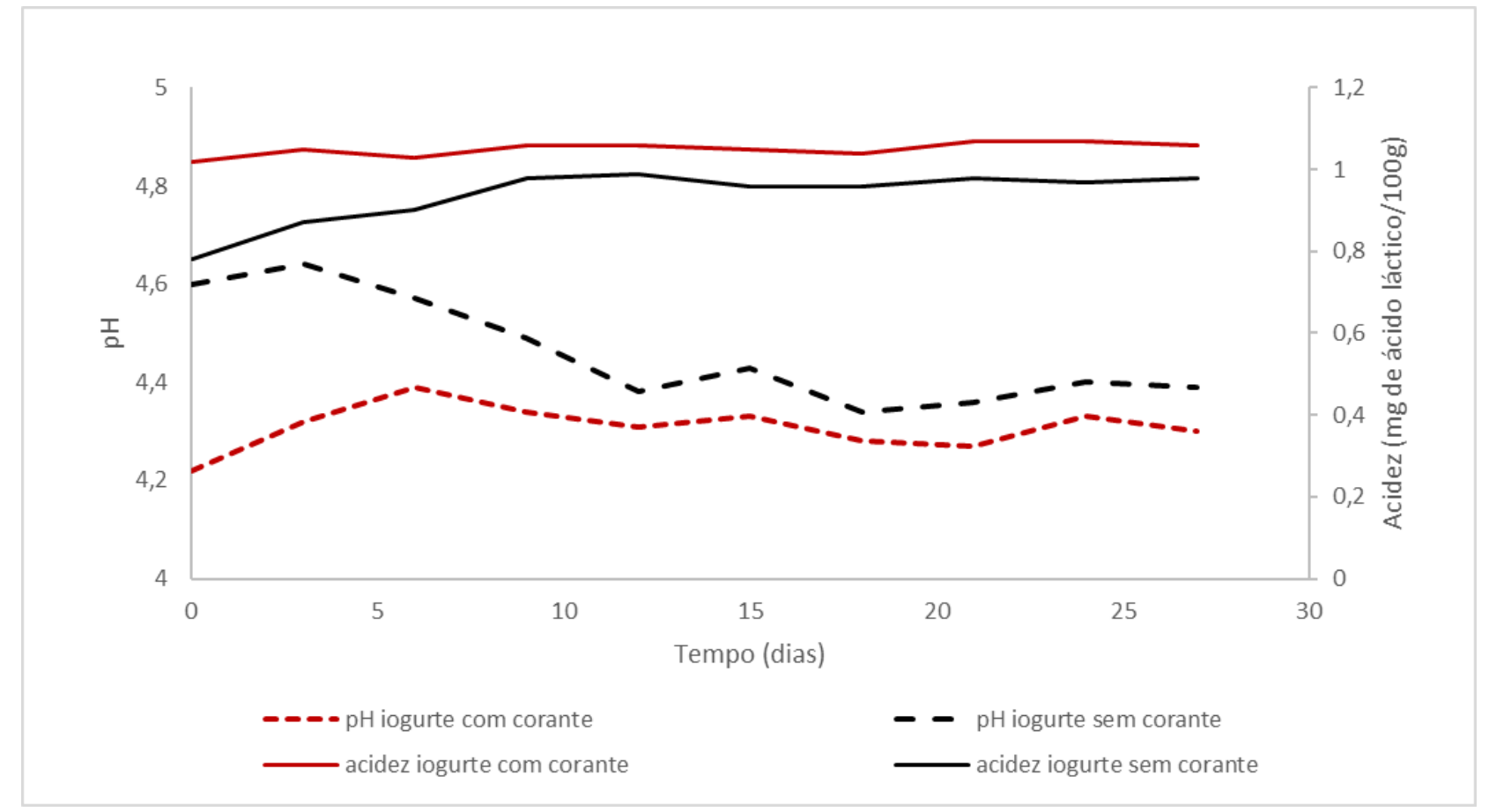

Fonte: Autores (2022).

A capacidade de retenção de água dos iogurtes se manteve durante o tempo de armazenamento, com regressão linear não significativa para os dois tratamentos utilizados (Figura 4). A estabilidade da matriz de gel é desejável ao iogurte por assegurar a qualidade sensorial do produto ao longo do armazenamento. Os resultados obtidos demostram que os sistemas de produção e armazenamento empregados foram satisfatórios, permitindo a manutenção das condições de temperatura, pH e ausência de fatores mecânicos, reconhecidos como os principais agentes ocasionadores da sinérese (Dias et al., 2020). Os resultados também demostraram que a adição de corante ao iogurte não causou nenhum prejuízo a esse parâmetro de qualidade. 
Figura 4. Capacidade de retenção de água de iogurtes sem corante e com corante natural obtido a partir de capim-gordura, ao longo do tempo de armazenamento.

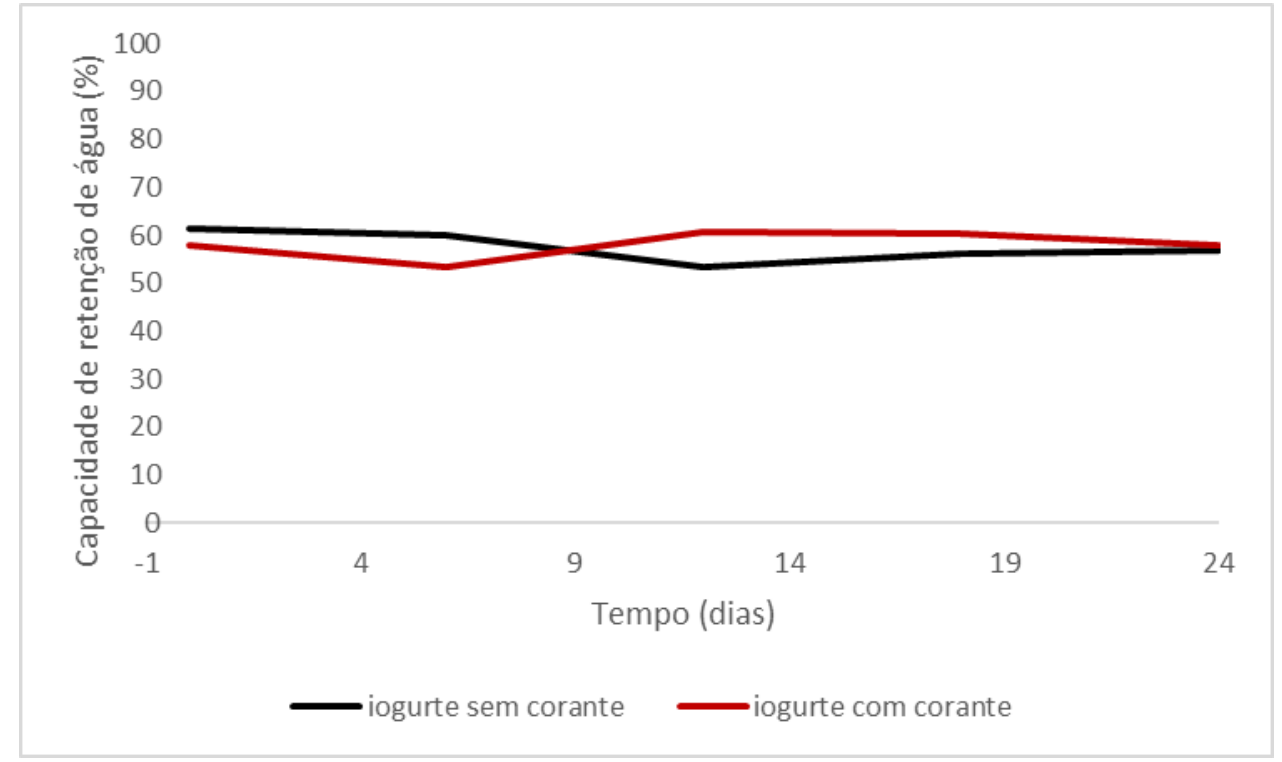

Fonte: Autores (2022).

A variação da cor $\left(\Delta \mathrm{E}^{*}\right)$ dos iogurtes adicionados de corante (Figura 5) é uma estimativa da mudança da coloração dos iogurtes ao longo do tempo de armazenamento, tendo como referência a coloração apresentada no primeiro dia. Fato esse, de real importância para o presente trabalho, uma vez que as antocianinas são compostos muito reativos e, por isso, muito instáveis, podendo ser facilmente degradadas ao longo do armazenamento. No entanto, os resultados obtidos demostram que a coloração do iogurte variou pouco ao longo do armazenamento, sendo de difícil percepção a olho nu $(\Delta$ inferior a 3,5) (Moura et al., 2019).

Figura 5. Variação da coloração $\left(\Delta \mathrm{E}^{*}\right)$ dos iogurtes adicionados de corante natural obtido a partir de capim-gordura ao longo do tempo de armazenamento.

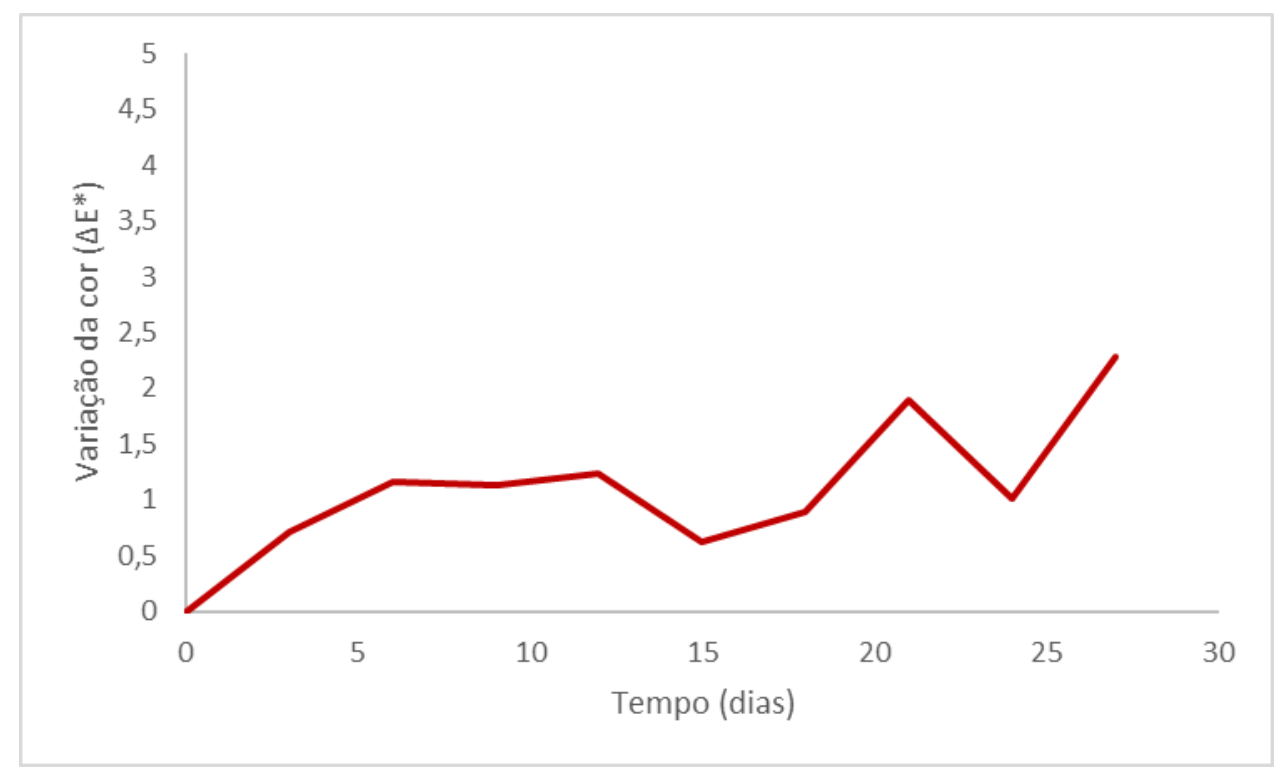

Fonte: Autores (2022). 
Ścibisz et al. (2019) estudaram as variações da coloração de iogurtes adicionados de morango, mirtilo e ginja (cereja ácida) ao longo de oito semanas de armazenamento. Os resultados demostraram que a concentração de antocianinas presentes nos iogurtes (oriundas dos frutos) reduziu ao longo do armazenamento, especialmente nas duas primeiras semanas. De acordo com os autores, as antocianinas presentes no iogurte de mirtilo foram as mais estáveis, provavelmente devido à presença de antocianinas aciladas. A menor estabilidade foi obtida para as antocianinas de morango, que, além de serem estruturalmente mais susceptíveis, podem ter tido sua degradação acelerada pela presença de ácido ascórbico nesse fruto. Além disso, a coloração desses iogurtes também foi afetada, com a tendência da diminuição da intensidade ao longo do armazenamento e a alteração da cor de vermelho para laranja, indicando uma provável degradação de antocianinas e/ou a formação de compostos polimerizados.

Resultados mais próximos aos encontrados nesse trabalho foram descritos por Moura et al. (2019) em iogurtes coloridos com extrato de hibisco microencapsulados. Os iogurtes apresentaram queda do teor de antocianinas ao longo do armazenamento, sendo essa menor com o emprego do encapsulamento. No entanto, as diferenças nas colorações $(\Delta)$ não foram superiores a 2,73, mesmo no extrato não encapsulado.

A estabilidade da cor apresentada pelos iogurtes nesse trabalho pode estar relacionada a fatores como estabilidade do pigmento extraído do capim-gordura, condições favoráveis de armazenamento, interações que proporcionaram maior estabilidade do pigmento.

As antocianinas presentes no capim-gordura são derivadas de cianidina, antocianidina não metilada (Figura 6). No entanto, algumas das formas apresentadas possuem diferentes graus de metilação da antocianina, conjugadas com a glicose, (malonil e metilmalonil), que podem contribuir significativamente para a estabilidade desses pigmentos (Neves et al., 2021). A metilação é capaz de aumentar a estabilidade de antocianinas à temperatura e exposição à luz, além de aumentar o seu potencial protetor antioxidante (Skates et al., 2018; Zhang et al., 2019).

Figura 6. Algumas das estruturas de antocianinas presentes no capim-gordura.

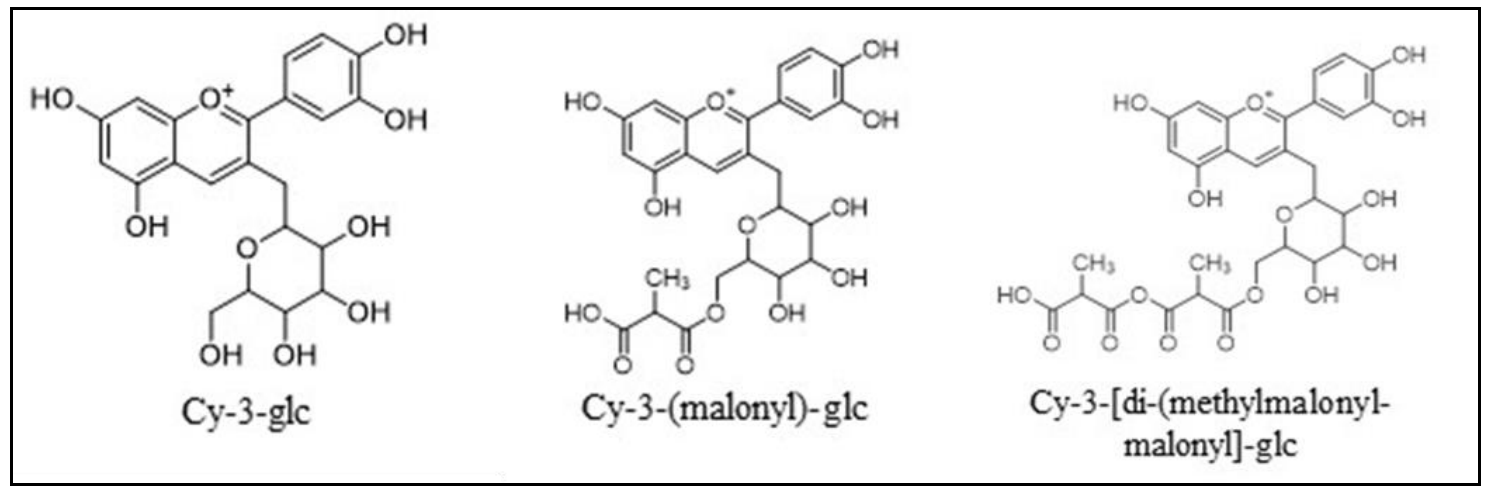

Fonte: Neves et al (2021) adaptado.

As antocianinas são compostos termo sensíveis e tendem a apresentar maior estabilidade a menores temperaturas. Em um estudo com antocianinas de mirtilo, Liu et al. (2018) concluíram que o aumento da temperatura aumenta a taxa de degradação das antocianinas, sendo que a taxa de preservação começa a declinar acentuadamente quando a temperatura ultrapassa $50^{\circ} \mathrm{C}$. No mesmo trabalho foi concluído que o $\mathrm{pH}$ também exerce forte efeito sob a estabilidade dos pigmentos, sendo que valores abaixo de 6 favoreceram a estabilidade das antocianinas de mirtilo.

Em outro trabalho, Rosa et al. (2021) concluíram que as temperaturas de refrigeração $\left(3^{\circ} \mathrm{C} \pm 4^{\circ} \mathrm{C}\right)$ e congelamento $(-$ $18^{\circ} \mathrm{C} \pm 5^{\circ} \mathrm{C}$ ) foram melhores para a preservação das antocianinas quando comparadas à temperatura ambiente. Assim, a temperatura de armazenamento dos iogurtes $\left(4^{\circ} \mathrm{C} \pm 1^{\circ} \mathrm{C}\right)$ bem como o $\mathrm{pH}$ característico desse produto (média de 4,31 para $\mathrm{o}$ 
iogurte com corante) podem ter contribuído para a estabilidade das antocianinas de capim-gordura adicionadas. A análise de antocianinas no iogurte ao longo do armazenamento poderia contribuir para o argumento, no entanto não foi possível realiza-la uma vez que a concentração encontrada nos iogurtes está abaixo do limite de detecção do método de pH diferencial, adotado nesse trabalho.

Além dos fatores citados, o próprio iogurte também pode ter contribuído para a manutenção da cor ao longo dos 27 dias de armazenamento. Segundo Ścibisz et al. (2019) a interação não covalente entre as antocianinas e a proteína do leite pode melhorar a estabilidade do pigmento. A interação entre antocianinas e proteínas também foi confirmada por Wang \& Xie (2019), que descreveram a interação de cianidina-3-glicosídeo com proteína de soja por vias hidrofóbicas e que o complexo formado aumentou a estabilidade do pigmento em temperaturas de 80 e $90^{\circ} \mathrm{C}$.

\section{Conclusão}

A presente pesquisa apresentou o capim-gordura como uma matéria-prima capaz de fornecer extratos ricos em antocianinas de maneira rápida e barata. A extração de antocianinas de capim-gordura foi otimizada com a utilização de solução de etanol a 38\% (v/v) em água e proporção de 1:3,33 (capim: solução extratora). A sonicação se mostrou mais eficiente do que a agitação para a extração. O processo de obtenção do corante natural a partir de antocianinas de capimgordura, com a utilização das etapas de evaporação e liofilização se mostraram viáveis.

A incorporação de corante natural de antocianinas extraídas de capim-gordura em iogurtes resultou em produtos com coloração persistente ao longo do armazenamento, mostrando a potencialidade dessa matéria-prima para a elaboração de corantes alimentícios. Embora com características diferentes do iogurte sem corante, a utilização do corante de capim-gordura não comprometeu a qualidade dos iogurtes quanto ao $\mathrm{pH}$, acidez e capacidade de retenção de água, mesmo depois de 27 dias de armazenamento.

O presente trabalho é um resultado preliminar bastante promissor que servirá de ponto de partida para futuros projetos onde poderão ser avaliadas as condições de toxicidade e segurança microbiológica dos iogurtes para a condução de um teste sensorial. Além disso, pode ser pesquisado outras formas de aplicação do corante, em outras matrizes alimentícias e diferentes condições de preparo e armazenamento de alimentos.

\section{Agradecimentos}

Os autores agradecem à Universidade Federal dos Vales do Jequitinhonha e Mucuri (UFVJM) e ao Instituto de Ciência e Tecnologia (ICT) pelo suporte institucional. Agradecemos à (FAPEMIG) Fundação de Amparo à Pesquisa do Estado de Minas Gerais pela concessão de bolsa de Iniciação Científica a I. M. Silva.

\section{Referências}

Albuquerque, B. R., Pinela, J., Barros, L., Oliveira, M. B. P. P., \& Ferreira, I. C. F. R. (2020). Anthocyanin-rich extract of jabuticaba epicarp as a natural colorant: Optimization of heat- and ultrasound-assisted extractions and application in a bakery product. Food Chemistry, 316, 126364. https://doi.org/10.1016/j.foodchem.2020.126364

Amchova, P., Kotolova, H., \& Ruda-Kucerova, J. (2015). Health safety issues of synthetic food colorants. Regulatory Toxicology and Pharmacology, 73(3), 914-922. https://doi.org/10.1016/j.yrtph.2015.09.026

Backes, E., Leichtweis, M. G., Pereira, C., Carocho, M., Barreira, J. C. M., Kamal Genena, A., C.F.R. Ferreira, I. (2020). Ficus carica L. and Prunus spinosa L. extracts as new anthocyanin-based food colorants: A thorough study in confectionery products. Food Chemistry, 333 , 127457. https://doi.org/10.1016/j.foodchem.2020.127457

Batista, R. V., Rosário, F. M., Alves, V., Francisco, C. T. dos P., Tormen, L., \& Bertan, L. C. (2020). Desenvolvimento de iogurte tipo "sundae" sabor jabuticaba (Myrciaria jaboticaba (Vell) Berg) com adição de fibras. Research, Society and Development, 9(9), e214996662. https://doi.org/10.33448/rsdv9i9.6662 
Brasil (2007) Ministério da Agricultura, Pecuária e Abastecimento. Instrução Normativa n 46, de 23 de outubro de 2007 . Adota o Regulamento Técnico de Identidade e Qualidade de Leites Fermentados, anexo à presente Instrução Normativa. Diário Oficial da República Federativa do Brasil, 18 out. 2007.

Carvalho, N. K. G. de, Leite, D. O. D., Camilo, C. J., Nonato, C. de F. A., Salazar, G. J. T., Costa, L. R., \& Costa, J. G. M. da. (2020). Perfil Químico e Análise Antioxidante in vitro do extrato e fração de Auxemma glazioviana Taub. Research, Society and Development, 9(10), e9699109387. https://doi.org/10.33448/rsd-v9i10.9387

Cho, W.-Y., Kim, D.-H., Lee, H.-J., Yeon, S.-J., \& Lee, C.-H. (2020). Quality characteristic and antioxidant activity of yogurt containing olive leaf hot water extract. CyTA - Journal of Food, 18(1), 43-50. https://doi.org/10.1080/19476337.2019.1640797

Coelho, F. J. O., Quevedo, P. S., Menin, A., \& Timm, C. D. (2009). Avaliação do prazo de validade do iogurte. Ciência Animal Brasileira, 10(4), 1155-1160.

Derringer, G., \& Suich, R. (1980). Simultaneous Optimization of Several Response Variables. Journal of Quality Technology, 12(4), 214-219. https://doi.org/10.1080/00224065.1980.11980968

Dias, J. G., Guedes, J., Monteiro, R. de S., Pinto, V. R., Gandra, K. M. B., Cunha, L. R. da, \& Pereira, P. A. P. (2020). Avaliação da estabilidade de iogurte concentrado salgado adicionado de orégano durante o armazenamento. Research, Society and Development, 9(10), e8999109322. https://doi.org/10.33448/rsdv9i10.9322

Feketea, G., \& Tsabouri, S. (2017). Common food colorants and allergic reactions in children: Myth or reality? Food Chemistry, 230, 578-588. https://doi.org/10.1016/j.foodchem.2017.03.043

Gregório, M. G., Brito, A. N. dos S. L. de, Oliveira, A. G. de, Mascarenhas, N. M. H., Paiva, F. J. da S., Medeiros Neto, M. S. de, \& Silva, L. P. F. R. da. (2020). Desenvolvimento e caracterização físico-química de iogurte tipo grego com adição de diferentes concentrações da compota de carambola (Averrhoa carambola). Research, Society and Development, 9(8), e861986484. https://doi.org/10.33448/rsd-v9i8.6484

Harte, F., Luedecke, L., Swanson, B., \& Barbosa-Cánovas, G. V. (2003). Low-Fat Set Yogurt Made from Milk Subjected to Combinations of High Hydrostatic Pressure and Thermal Processing. Journal of Dairy Science, 86(4), 1074-1082. https://doi.org/10.3168/jds.S0022-0302(03)73690-X

Instituto Adolfo Lutz (IAL) (2008). Métodos físico-químicos para análise de alimentos. (O. Zenebon, N. S. Pascuet, \& P. Tiglea, Orgs.). São Paulo: Instituto Adolfo Lutz.

Jaekel, L. Z., Schmiele, M., \& Chang, Y. K. (2020). Impactos do amido resistente e da enzima transglutaminase nas características tecnológicas de espaguete. Research, Society and Development, 9(8), e891986219. https://doi.org/10.33448/rsd-v9i8.6219

Liu, Y., Liu, Y., Tao, C., Liu, M., Pan, Y., \& Lv, Z. (2018). Effect of temperature and pH on stability of anthocyanin obtained from blueberry. Journal of Food Measurement and Characterization, 12(3), 1744-1753. https://doi.org/10.1007/s11694-018-9789-1

Martins, N., Roriz, C. L., Morales, P., Barros, L., \& Ferreira, I. C. F. R. (2016). Food colorants: Challenges, opportunities and current desires of agroindustries to ensure consumer expectations and regulatory practices. Trends in Food Science \& Technology, 52, 1-15. https://doi.org/10.1016/j.tifs.2016.03.009

Meng, J., Kang, T.-T., Wang, H.-F., Zhao, B.-B., \& Lu, R.-R. (2018). Physicochemical properties of casein-dextran nanoparticles prepared by controlled dry and wet heating. International Journal of Biological Macromolecules, 107, 2604-2610. https://doi.org/10.1016/j.ijbiomac.2017.10.140

Mojica, L., Berhow, M., \& Gonzalez de Mejia, E. (2017). Black bean anthocyanin-rich extracts as food colorants: Physicochemical stability and antidiabetes potential. Food Chemistry, 229, 628-639. https://doi.org/10.1016/j.foodchem.2017.02.124

Moura, S. C. S. R., Schettini, G. N., Garcia, A. O., Gallina, D. A., Alvim, I. D., \& Hubinger, M. D. (2019). Stability of Hibiscus Extract Encapsulated by Ionic Gelation Incorporated in Yogurt. Food and Bioprocess Technology, 12(9), 1500-1515. https://doi.org/10.1007/s11947-019-02308-9

Neves, N. A., Stringheta, P. C., Gómez-Alonso, S., \& Hermosín-Gutiérrez, I. (2021). Anthocyanin Composition of Melinis minutiflora Cultivated in Brazil. Revista Brasileira de Farmacognosia, 31(1), 112-115. https://doi.org/10.1007/s43450-021-00133-w

Neves, N. A., Stringheta, P. C., Silva, I. F. da, García-Romero, E., Gómez-Alonso, S., \& Hermosín-Gutiérrez, I. (2021). Identification and quantification of phenolic composition from different species of Jabuticaba (Plinia spp.) by HPLC-DAD-ESI/MSn. Food Chemistry.

Ogliari, R., \& Novello, D. (2021). Avaliação de iogurte de coco baseado na perspectiva do consumidor: influência das informações do produto sobre o perfil sensorial. Research, Society and Development, 10(2), e53210212582. https://doi.org/10.33448/rsd-v10i2.12582

Pinela, J., Prieto, M. A., Pereira, E., Jabeur, I., Barreiro, M. F., Barros, L., \& Ferreira, I. C. F. R. (2019). Optimization of heat- and ultrasound-assisted extraction of anthocyanins from Hibiscus sabdariffa calyces for natural food colorants. Food Chemistry, 275, 309-321. https://doi.org/10.1016/j.foodchem.2018.09.118

Piovesan, N., Borges, M. F. de A., Queiroz, J. M. de, Souza, R. L. A. de, Oliveira, E. N. A. de, Oliveira, G. S., \& Oliveira, P. V. C. de. (2021). Caracterização físico-química e sensorial de frozen iogurte de kiwi enriquecido com prebiótico. Research, Society and Development, 10(8), e5710817031. https://doi.org/10.33448/rsd-v10i8.17031

Rodrigues, A. P. de S., Santos, G. Á. de S., Carvalho, H. J. M., Schmiele, M., Sobrinho, P. de S. C., \& Rocha, L. de O. F. (2021). Efeito da adição de colágeno e sucralose nas características tecnológicas e sensoriais do iogurte. Research, Society and Development, 10(11), e507101119841. https://doi.org/10.33448/rsdv10i11.19841

Rosa, J. R., Weis, G. C. C., Moro, K. I. B., Robalo, S. S., Assmann, C. E., Silva, L. P., \& Rosa, C. S. (2021). Effect of wall materials and storage temperature on anthocyanin stability of microencapsulated blueberry extract. $L W T, 142,111027$. https://doi.org/10.1016/j.1wt.2021.111027

Ścibisz, I., Ziarno, M., \& Mitek, M. (2019). Color stability of fruit yogurt during storage. Journal of Food Science and Technology, 56(4), 1997-2009. 
Research, Society and Development, v. 11, n. 3, e9811326230, 2022

(CC BY 4.0) | ISSN 2525-3409 | DOI: http://dx.doi.org/10.33448/rsd-v11i3.26230

https://doi.org/10.1007/s13197-019-03668-y

Silva, A. B. N. da, \& Ueno, M. (2012). Avaliação da viabilidade das bactérias lácticas e variações da acidez titulável em iogurtes com sabor de frutas. Revista do Instituto Latic. "Cândido Tostes”, 390(68), 20-25.

Silva, D. C. G. da, Abreu, L. R., \& Assumpção, G. M. P. (2012). Addition of water-soluble soy extract and probiotic culture, viscosity, water retention capacity and syneresis characteristics of goat milk yogurt. Ciência Rural, 42(3), 545-550.

Silva, F. C. G. da, Dalaqua, S., Azevedo, E. C. de, Campos, G. M. de, Raghiante, F., \& Martins, O. A. (2016). Profile of lactic acid within validity of full natural yogurt. Revista Brasileira de Higiene e Sanidade Animal, 10(4). https://doi.org/10.5935/1981-2965.20160050

Skates, E., Overall, J., DeZego, K., Wilson, M., Esposito, D., Lila, M. A., \& Komarnytsky, S. (2018). Berries containing anthocyanins with enhanced methylation profiles are more effective at ameliorating high fat diet-induced metabolic damage. Food and Chemical Toxicology, 111, 445-453. https://doi.org/10.1016/j.fct.2017.11.032

Vianna, F. S., Canto, A. C. V. da C. S., Costa-Lima, B., Salim, A. P., Balthazar, C. F., Costa, M. P., \& Silva, A. C. de O. (2019). Milk from different species on physicochemical and microstructural yoghurt properties. Ciência Rural, 49(6). https://doi.org/10.1590/0103-8478cr20180522

Wang, C., \& Xie, Y. (2019). Interaction of Protein Isolate with Anthocyanin Extracted from Black Soybean and Its Effect on the Anthocyanin Stability. Journal of Food Science, 84(11), 3140-3146. https://doi.org/10.1111/1750-3841.14816

Zhang, B., Jiang, X., Huang, G., Xin, X., Attaribo, T., Zhang, Y., \& Gui, Z. (2019). Preparation and characterization of methylated anthocyanins from mulberry fruit with iodomethane as donor. Sericologia, 59, 106-116. 